\title{
Service-Oriented Architecture-Guided Information Service System for Design and Implementation of Rural Tourism
}

\author{
Yukun You \\ Yantai Institute of Technology, Yantai, Shandong 264005, China \\ Correspondence should be addressed to Yukun You; youyukun@yitsd.edu.cn
}

Received 14 December 2021; Revised 2 January 2022; Accepted 3 January 2022; Published 25 January 2022

Academic Editor: Xin Ning

Copyright ( 2022 Yukun You. This is an open access article distributed under the Creative Commons Attribution License, which permits unrestricted use, distribution, and reproduction in any medium, provided the original work is properly cited.

\begin{abstract}
Rural tourism has become a way for people to pursue a simple life and relax, thanks to the variety of tourism forms available. Rural tourism, on the other hand, lacks a unified information platform for providing services to users and is unable to provide humanized services based on tourism experience. Rural tourism around the city has become the main force of tourism recovery because outbound tourism has yet to begin and domestic interprovincial tourism has only recently recovered. The development of rural tourism can be aided by an information service system that can effectively improve the utilization efficiency of tourism resources and explore new and reasonable paths for the development of rural tourism. This paper examines the opportunities and challenges that rural tourism faces in the face of the COVID-19 epidemic, as well as actionable strategies for the rapid growth of rural tourism. This paper introduces the Service-Oriented Architecture (SOA) technology required in the construction of the tourism service information sharing platform as the research object. In addition, the rural tourism information service system was established. So that rural tourism can be at the forefront of tourism's rapid recovery and revitalization, it should be promoted to become more vibrant.
\end{abstract}

\section{Introduction}

Particularly under the Rural Revitalization Strategy, rural tourism development takes into account the role of rural industrial structure adjustment and has become a new growth point to promote rural economic development [1]. Driven by national policies, China's rural tourism and leisure agriculture have developed rapidly, and the market scale of rural tourism industry has been expanding, which has become an important carrier of rural industrial integration [2]. With the rapid and vigorous development of economy, society, and tourism, due to the lack of timely, efficient, and rich technical methods, the traditional tourism information service management has some problems, such as incomplete tourism information, lack of vividness, and out-of-control flow of hot scenic spots, and tourists' requirements for tourism personalization, autonomy, and experience are increasing [3]. At present, some villages have not been effectively developed due to lack of rural tourism publicity and display platform and lack of detailed and practical rural road traffic navigation map and convenient rural tourism services, and there is a lack of horizontal and vertical organic combination between tourist attractions and tourism enterprises, which has been in a loose and fragmented state. Therefore, large-scale tourism has not been formed and has not been able to bring due economic benefits [4]. In order to display the unique rural tourism resources to tourists, attract tourists to the countryside to experience simple folk customs, beautiful natural beauty, long history, and culture and humanistic atmosphere, and improve the economic, ecological, and cultural benefits brought by rural tourism, it is urgent to establish a rural tourism information service system.

With the development of education, economy, and culture, people's tourism demand is also developing in a multilevel direction, which puts forward higher requirements on how to quickly and accurately obtain, process, and use tourism service information. Therefore, studying the key technologies of building tourism service information sharing platform will inject new vitality and power into the further 
development of tourism service informatization [5]. We should actively promote the development of rural tourism information services in the intelligent era, improve the intelligent level of tourism management, systematically integrate rural tourism resources, develop a large number of new rural tourism products, tap the development potential of rural tourism, and provide intelligent, efficient, and humanized tourism services for the majority of tourists [6]. A component model is Service-Oriented Architecture (SOA). SOA features loose coupling and platform independence, allowing businesses to add new services or update existing services in a modular fashion to meet new business needs and adapt to changes [7]. People's lives continue to change and remain unchanged, and tourism has progressed from flow restriction within the region to the recovery stage of interprovincial and regional team tourism, which undoubtedly provides strong policy support for the revitalization and development of tourism [8]. The goal of this paper's research is to assist rural areas in establishing a "rural tourism information service system based on SOA" that can aid in the development of rural tourism.

After these years of development, there are as many tourism information service networks, and the development of these websites is becoming more and more mature $[9,10]$. From the cultural attribute of tourism resources, rural tourism is a kind of tourism activity that relies on rural tourism resources and feels and experiences rural folk cultural life. Relatively speaking, rural tourism is more authentic and regressive than urban tourism [11]. In the postepidemic period, rural tourism will inevitably attract more and more tourists with its characteristics of simplicity, nature, and health and safety so as to meet the psychological needs of tourists for outdoor hiking, getting close to nature, emotional catharsis, and spiritual healing. Starting from the value of rural tourism and the development characteristics in the postepidemic period, this paper puts forward the development countermeasures of rural tourism in the postepidemic period and establishes the rural tourism information service system. SOA is applied to the tourism service information sharing platform through Web Service technology, which effectively solves the information sharing and collaborative work among tourism-related enterprise systems in tourism destination cities and improves the overall service level. Rural tourism should be promoted to glow with greater vitality.

\section{Related Work}

Literature $[12,13]$ proposed that "SOA" is the most important topic in the field of modern application development. Literature [14] constructs a tourism service information sharing platform based on SOA, which can meet the needs of tourists to quickly obtain all-round tourism information services of tourism destinations before tourism (e.g., choose tourist attractions, understand tourist routes, means of transportation, service providers, catering and accommodation, weather temperature, etc.). Literature [15] discusses the typical construction framework and key implementation technologies of SOA and analyzes and summarizes the characteristics, elements, and roles of SOA. Literature [16] studies and designs in detail the implementation and key technologies of the presentation layer, business logic layer, and data persistence layer in the tourism service information sharing platform. It also realizes the information sharing among scenic spots, public transportation, and hotels. Literature [17] holds that rural tourism can be divided into traditional rural tourism and modern rural tourism. Literature [18] holds that modern rural tourism is different from traditional rural tourism. Modern rural tourism is a tourism model in rural areas. It is a new rural tourism model based on rural tourism resources such as unique beautiful scenery, natural environment, historical buildings, and long history and culture. Literature [19] proposes that service is a process in which service providers deliver content to service objects through certain channels. Design is a kind of creative behavior that gathers the elements of each part for a certain purpose and function and considers the overall effect. The combination of service and design is a relatively new concept in the research of modern design. Service design mainly studies the systematic application of design theories and methods to the creation, definition, and planning of services. Literature [20] holds that rural tourism is an attempt to extend modern tourism to traditional agriculture. Through the promotion of tourism, it organically integrates ecological agriculture and ecotourism, which is a new industrial form. Literature [21] proposes that rural tourism can be combined with orchards, tea gardens, gardens, leisure fishing grounds, agricultural education, agricultural science popularization demonstration, customs and folk customs, fitness and recuperation, and fitness and entertainment so as to broaden horizons and develop in an all-round way. Literature [22] believes that rural flavor is the center and unique selling point of rural tourism. Literature [23] believes that we should pay attention to the experience elements of tourists so as to build the relevant discipline system. Literature [24] believes that the application of information technology is very important to the development of rural smart tourism. At present, the tourism industry in some areas has adopted the information operation and management mode, and rural tourism has also actively adapted to the requirements of new technology and the development of information society. However, at present, the information construction in many villages is not perfect, and there are some disadvantages. Literature [25] puts forward that the main application aspects of tourism service informatization are the application of the latest development technology of information sharing in tourism information system. This paper discusses the related theoretical knowledge and key implementation technologies of SOA, as well as the application technology and thinking mode of information service system, and combines 3D simulation with 360-degree omnidirectional real-life display. It integrates various service modes such as rural tourism resource display, rural tourism marketing platform, and rural tourism itinerary planning. And every effort is made to create an all-round innovative new model of rural tourism service. 


\section{Methodology}

3.1. The Necessity and Problems of Rural Tourism Information Service Construction. Under the current social development background, the policy of rural revitalization has been further optimized, and China has paid full attention to the policy of rural revitalization, and the policy of rural revitalization has been regarded as the cooperative development of many industries in building a well-off demonstration village in an all-round way in China, and the industrial development has driven the economic income of the whole rural population to be effectively increased so as to achieve the purpose of rural revitalization. Under the optimized background of rural revitalization development model, it is necessary to further build the whole rural tourism informatization.

In the process of providing tourism information, I am eager to create an applicable sharing platform for tourism service information so that all service providers of tourismrelated enterprises can obtain the necessary information at any time and from any location, thereby supporting highquality tourism information service and enabling tourists to master and obtain as many effective service information as possible in the tourist destination cities they will visit [26]. The main goal of constructing a tourism information sharing platform is to raise the level of tourism information service, increase tourists' efficiency in obtaining useful information, and promote the quality of tourism information service providers' services through the network. As a result, in order to adapt to the network society, it is necessary to develop a practical tourism service information sharing platform model.

People's long-term travel needs have not been met as a result of the epidemic situation, and the backlog of inner emotions and emotions has reached a peak, and all they need now is a place of spiritual comfort to express their emotions and relieve their stress. Rural tourism, with its peaceful natural setting and simple rural flavor, satisfies people's emotional yearnings to reconnect with nature. Furthermore, the epidemic has not completely faded from people's lives, and rural tourism can better ensure the safety and health of tourists' travel by focusing on product supply and location conditions. Tourists who pay attention to their tourism experience not only passively perceive, acquire, and use network information, but also realize timely feedback service evaluation and popular supervision of market behavior norms through information evaluation systems, allowing tourists to contribute to the development of tourism service platform systems.

At present, social information technology is changing with each passing day, and information technology plays a great role in effectively utilizing tourism resources and improving the distribution and use of tourism resources and infrastructure [27]. Tourism information management and public information service improve the travel efficiency and experience of tourists and play an irreplaceable role in promoting the healthy development of tourism. Fully applying the information-based management mode can further highlight the innovative features of China's rural tourism in the current era. And the scale of the rural tourism market can be effectively expanded, and the experience of the whole rural tourism participants can be effectively enhanced. It can effectively transform the traditional rural tourism service mode and further optimize the corresponding tourism information construction path. Tourists can fully understand a lot of information about rural tourism through the information platform and make subjective choices of their own tourist destinations by means of information.

With the continuous development of information technology, more and more industries begin to attach importance to information construction, hoping to innovate the development path of industries through integration with information technology. Tourism informatization construction should first change the traditional management concept and apply informatization to all aspects of rural tourism construction. However, rural tourism resources are generally small in scale and scattered in different regions. However, the development of rural tourism is short, and it is still in the period of continuous exploration and improvement. The information and data resources themselves are not perfect, which cannot meet the needs of tourism informatization construction.

It is necessary to fully and effectively improve the traditional management concept and apply the specific informatization management mode to the rural tourism construction process during the process of rural informatization tourism construction. To fully and effectively integrate smart tourism, it is necessary to improve the infrastructure of rural tourism as a whole. Rural tourism, on the other hand, involves many departments and industries that are independent of one another, lack unified information sharing channels, and are unable to achieve interconnection, all of which add to the difficulty of building tourism informatization. Furthermore, due to an insufficient level of regional economic development, a lack of correct understanding of tourism informatization construction, and a lack of awareness of the impact of tourism informatization on the traditional business model and the change of the future rural tourism development model, the construction of tourism informatization infrastructure is lagging, receiving insufficient attention from the government. As a result, the construction of tourism informatization infrastructure is lagging, receiving insufficient attention from the government.

Tourists have developed the habit of using big data to screen, query, and understand tourist attractions in the context of a mature application of intelligent information technology. Local farmers and entrepreneurs, on the other hand, are the majority of the developers and operators of rural tourism. Tourism informatization's importance is determined by the quality and profitability of rural tourism. The development cost of rural tourism development operators is a constraint. Tourism marketing is primarily done through traditional advertising. They are unable to grasp network groups and attract them. There are few rural tourism publicity websites in Baijian, and rural tourism network publicity is primarily dependent on large-scale tourism websites, which primarily focus on the introduction of tourism resources and the lack of network e-commerce 
services. The established tourism websites are ineffective in terms of management, publicity, and marketing, and they have become "information islands."

3.2. System Integration Technology SOA. Tourism, as a sunrise industry, an industry that will never be eclipsed, is full of vitality. However, due to the emergence of COVID-19 epidemic, it has brought a great impact on rural tourism. The information system is developing faster and faster, and the business functions are becoming more and more complex. Due to various reasons, the development languages and technical systems of each system are different, which requires the application of appropriate system integration methods and technologies to integrate heterogeneous systems and data. At present, the widely used information system integration method is the system integration method of SOA. SOA is a distributed application architecture. In this architecture, the basic organizational elements are a group of relatively independent services. These services interact with each other through well-defined interfaces and calling protocols based on message mechanism, thus forming an application system. Among them, service is a set of functions that are clearly defined, self-contained, and independent of context or other services. Services are the units of application logic, and they provide a message-based interface suitable for accessing through the network.

The rural tourism information service system has many complicated subsystems. Because of the special requirements of each system for data processing and operating systems, the operating systems used by each subsystem are not the same, and the databases used by each subsystem are not completely unified. Rural tourism information service system is a comprehensive and integrated information system. Service has considered the reuse problem at the time of development and provides a standard interface, which can be called by various applications and other services. Therefore, through SOA architecture, services can be independently designed and developed, and when necessary, services can be integrated to become a part of the application. The typical implementation architecture of SOA is shown in Figure 1.

Service design is similar to system design in that it focuses on the integrity and smooth operation of the service system, as well as effectively organizing and planning the people, infrastructure, communication methods, and materials involved in the service project so that service providers can provide better user experience and service quality to the recipients. We must adhere to the people-oriented concept throughout the entire design process. The goal of the design is to meet the actual needs of the users. In order to tap into users' potential needs, we must pay attention to their sense of participation in order to obtain timely and accurate feedback information from them, and then improve the design content over time until their needs are met. The architecture of integration of rural tourism informatization service system based on SOA is proposed as shown in Figure 2 based on the needs of integration of each subsystem of rural tourism informatization. The user layer, service layer, business layer, and data layer are the four layers that make up the system. It solves the problem of data sharing and information communication among rural tourism information service subsystems.

In reality, when users of rural tourism information system use a certain function, a certain service, or complete a certain task, they may need data transmission and information sharing among the systems and cooperative operation among the systems. If a complex task is completely completed by a subsystem independently, there will be overlapping parts of each subsystem in the process of construction, which will result in waste of resources and redundancy of the system, and it is not convenient to maintain information and data. Web Services is a SOAcompliant technology. Web Services provides services through standard Web protocols. Its main purpose can be said to be the greatest feature that it can support interoperability between application service programs of different systems.

3.3. System Establishment. The first stage of system development is system analysis. Its job is to communicate with users, accurately analyze their needs, and determine which problems and tasks computers should solve. Even if the design and coding work is well done, if the platform requirement analysis is not detailed, comprehensive, and indepth, the system will not truly meet the needs of users, will not be well applied and popularized, and will not bring real economic benefits and use value. When tourists arrive at their destination, their primary concern is whether they will arrive on time. As a result, when choosing a tourist route, try to take the route that takes the least amount of time and has the shortest journey possible, while also considering the best route that transfers vehicles and costs the least amount of money. In reality, however, achieving all three goals at the same time is difficult. As a result, this paper draws lessons from the idea of breadth first with higher efficiency in the process of improving the bus query algorithm for tourist destinations. The basic idea is to search all of the tourist stops each time, then add all of the unexplored neighbors to the search queue, and repeat until the queue is empty.

Tourist transportation modes are divided into trains, cars, planes, and water transportation based on different spatial routes and carrying modes. Road transportation is the most flexible and convenient option among them, and it is the most popular among tourists. Cars, tour buses, electric cars, and bicycles are some of the modes of transportation available. Trains and high-speed trains have become important travel tools as China's railway infrastructure has developed and matured. Air and water transportation provide services in specific areas or longdistance transportation, and traffic ontology can be subdivided into price, distance, safety, and punctuality, which tourists can use as restriction conditions to choose from, thus providing tourists with more suitable modes of transportation.

Using the idea of breadth priority to find out the feasible routes between any two bus stations, you can query and 

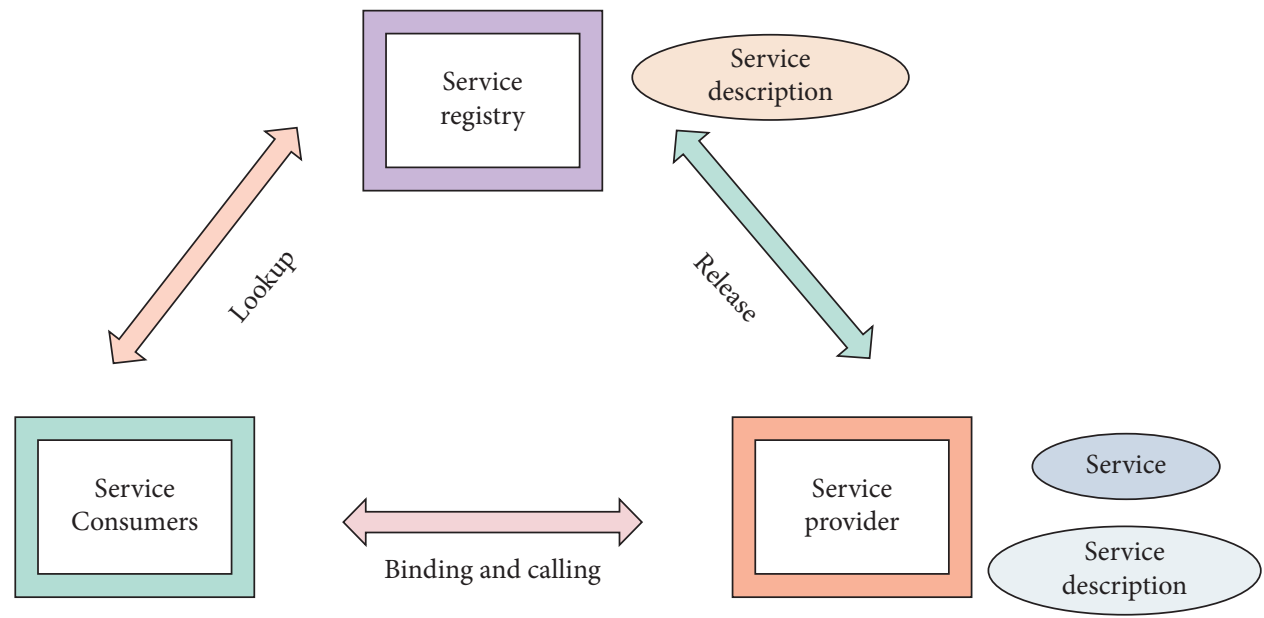

FIgURE 1: Typical implementation architecture of SOA.

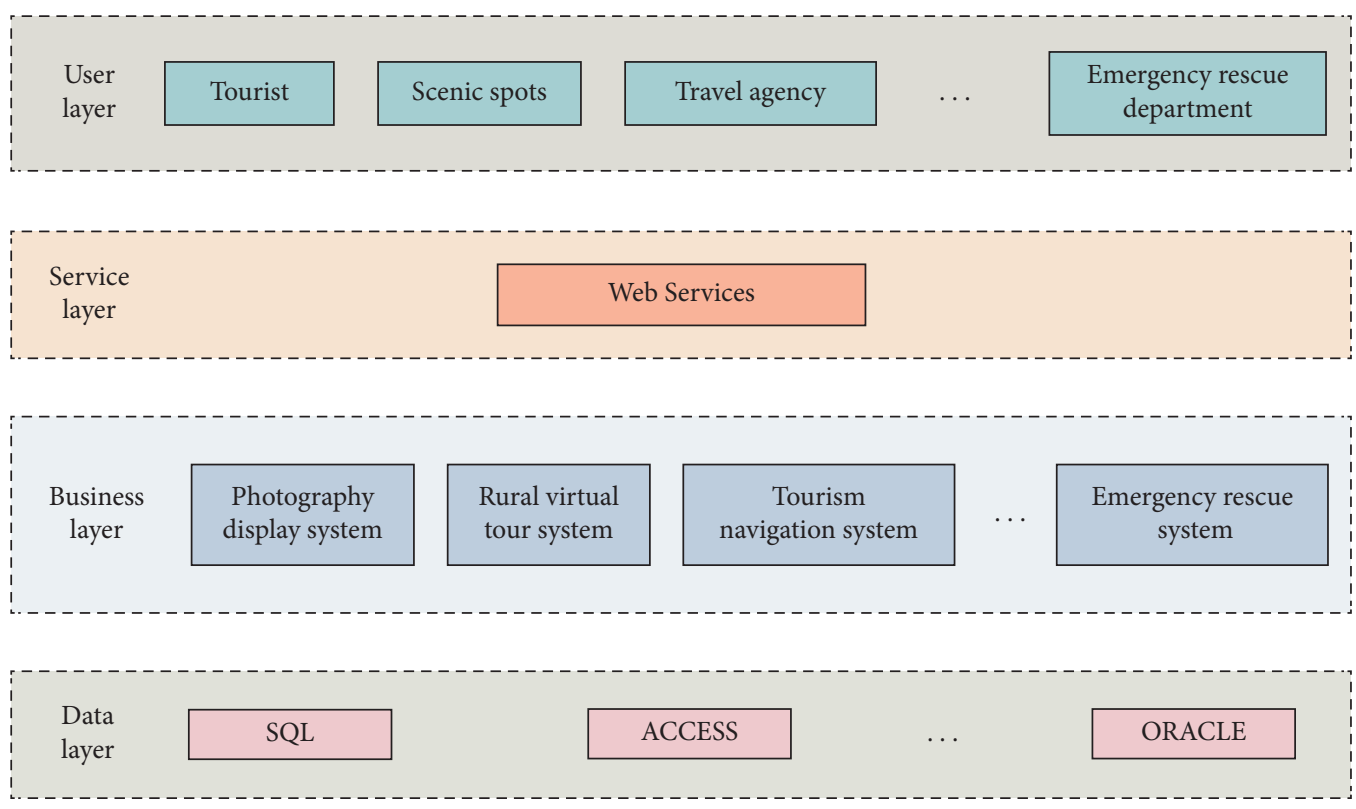

FIGURE 2: The architecture of rural tourism information service system integration based on SOA.

select these routes according to different needs so as to find out the optimal route that meets your query requirements.

(1) When the average travel time of adjacent bus stations is the same, the average time for bus transfer is the same, and all buses are running normally; the shortest time is taken as the optimal route model. Total travel time $T_{k}$ is equal to the sum of travel time and transfer time:

$$
\begin{aligned}
\operatorname{Min} T_{k} & =t_{a} \times \sum_{m=1}^{C 1_{k}+1}\left(G Z_{k, m}-1\right)+t_{b} \times C 1_{k}, \\
m & =1,2, \ldots, C 1_{k}+1 ; \quad k=1,2, \ldots, \tilde{k} .
\end{aligned}
$$

Among them, $t_{a}$ represents the average travel time of neighboring bus stations, $t_{b}$ represents the average time spent on bus transfers, and the $k$ th route is one or more of the above transfer routes.

(2) The model with the least number of transfers as the optimal route:

$$
\operatorname{Min} C 1_{k} \text {. }
$$

This model is equivalent to the above transfer routes according to the priority order of direct, one transfer, and two transfers.

(3) The model with the least cost as the optimal route:

$$
\operatorname{Min} Y_{k}=\sum_{m} Y X_{k, m}
$$

Through the preliminary demand investigation of the subject and object of rural tourism, the rural tourism 
information service system can be divided into three major systems, namely, rural tourism resource display system, rural tourism public service system, and rural tourism product marketing system. Secondly, the three major systems of rural tourism information service system are further refined, and the rural tourism resources display system can be divided into photography works display system and rural tourism virtual tour system. Rural tourism public service system is divided into rural tourism comprehensive information management system, tourism route recommendation and customization system, rural tourism navigation system, and emergency rescue system. Tourism marketing system is divided into tourism product information release system, online booking system, and online payment system. Man, machine, and environment are important components in the system. System design should not only study their characteristic elements, but also deeply study the relationship among them and analyze the construction principles and design methods of service system from a systematic point of view.

From the application point of view, the tourism information sharing platform is very object-oriented, and all users who need tourism information services can apply to access and share information resources. If these users are divided, they can be divided into three categories: tourismrelated enterprises, government tourism authorities, and tourists. Tourism, as a service industry, whether it is the government's tourism authorities or tourism-related enterprises, ultimately they all serve tourists.

Divide the rural tourism data sample data set $W$ into training set $A$ and test set $B, W=A \cup B$. When constructing the prediction model, continue to select the prediction subset $C$ so that

$$
W=A \cup B \cup C
$$

To construct the relationship between the output $y$ of the prediction model and the $x_{1}, x_{2}, \ldots, x_{n}$ input function, the Kolmogorov-Gabor polynomial is as follows:

$$
y=f\left(x_{1}, x_{2}\right)=a_{0}+a_{1} x_{1}+a_{2} x_{2}+a_{3} x_{1}^{2}+a_{4} x_{2}^{2}+a_{5} x_{1} x_{2} .
$$

Consider different monomials as $m$ input models in the initial system of the modeling network:

$$
v_{1}=a_{0}, v_{2}=a_{1} x_{1}, v_{3}=a_{2} x_{2}, \ldots, v_{6}=a_{5} x_{1} x_{2} .
$$

Build the first-level intermediate model:

$$
z_{k}=f_{k}\left(v_{i}, v_{j}\right), \quad i=1,2, \ldots, 6 .
$$

In the training set $A$, the coefficient of $z_{k}$ is obtained with the help of the parameter prediction model. In the test set $B$, the competitive model $\left\{z_{k}\right\}$ is filtered, and the intermediate candidate prediction model $w_{k}=\left(z_{k}\right)$ is collected and regarded as the input of the second layer of the network.

The various subsystems are fully, effectively, and reasonably integrated, based on the platform's own application integration and integration capabilities, through unified identity authentication integration, web interface integration, data exchange integration, and other integration technologies, to provide rural tourists, farmers, and merchants. Users who are interested in rural tourism can access a wide range of information. The platform's functional requirement analysis is completed, and various functional modules are designed using platform design methods and principles. Focus is on the tourist bus travel module's design, which includes the humanized creation of query standards, as well as the improvement of the bus travel transfer algorithm and the bus and subway simultaneous travel transfer algorithm.

\section{Result Analysis and Discussion}

Under the unexpected events such as the COVID-19 epidemic, people's demand preference for tourism has changed qualitatively, and tourists have changed from high-level needs such as social interaction, self-esteem, and identity to low-level needs such as safety and hygiene, which is driven or forced by the infectious characteristics of the epidemic. The change of demand will change people's preference for the choice of tourism destinations and then affect the behavior of tourism decision makers. Rural tourist destinations are relatively empty, mainly outdoor activities, and there is a certain distance between people, which meets the needs of tourists for safe travel at present, is not easy to cause the spread of epidemic, and is the best choice for tourists at present.

The user experience model's design elements are adaptable and comprehensive, and they will be updated and improved over time as space and time evolve. People's methods of obtaining tourism information have changed as a result of the development of the mobile Internet. Tourists frequently use the mobile Internet to obtain food, lodging, transportation, travel, shopping, entertainment, and other information. Various mobile tourism information service platforms are constantly emerging, and these platforms offer corresponding services for various user needs. The degree of data sharing among service platforms, however, is low due to enterprises' pursuit of market economic benefits. At the same time, there has been no comprehensive study of tourists' experiences, resulting in the fact that the information service platform only offers pretravel inquiry and purchase, as well as posttravel sharing and evaluation services, ignoring tourists' travel needs. The information platform's network information is numerous and disorganized, lacking in timeliness, accuracy, and systematization. Compare the results of two different preprocessing methods and test the model's performance on the data set. The performance is shown in Figure 3.

The effective solution is to build a basic comprehensive information platform on the technical level based on various existing tourism information service systems and integrate the existing tourism information service data and business functions in various information systems into the platform. According to the preference settings in the scenic spot classification model and tourist model, the system can more accurately recommend the scenic spot information that tourists are interested in and meet the needs of personalized access to information and services. Figure 4 shows the comparison of the performance time of the two algorithms. 


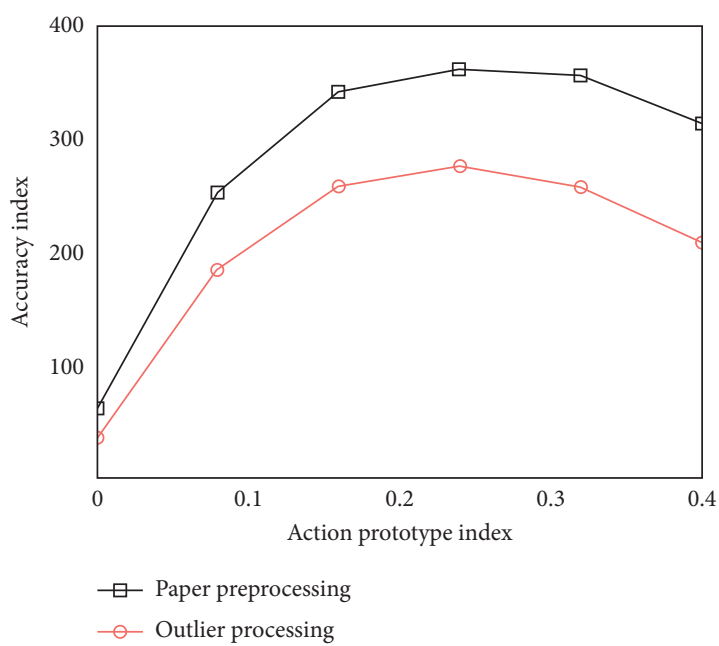

Figure 3: Comparison of accuracy obtained by different pretreatment methods.

There are a lot of GIS geospatial data on the server, and there are a lot of visits, so the system is under great pressure. It is planned to adopt the mode of dual-computer hot standby and disk display. The core switching layer, which is composed of two high-end multilayer switches, can realize fault-tolerant work of two computers and ensure high-speed data exchange. Figure 5 shows the comparison of accuracy and recall of different methods.

Because of the difference of users' preferences, the service system should automatically identify users' preferences by corresponding recommendation algorithms, combining the contextual information of tourists and tourism products, so as to provide users with personalized and functional customized recommendation content and realize timely access to tourism information with characteristic needs.

Realize digitalization, standardized management, and efficient utilization of rural tourism resource information using database technology, virtual tour technology, and other technologies. Analyze, sort out, and deeply develop information resources related to rural tourism, create a core database of rural tourism resource information, implement distributed management of rural tourism information, and provide basic data support for rural tourism information integration service. The systematic and organized excavation of tangible and intangible contact points with various roles and dimensions, resulting in service value, is known as service design. It entails a wide range of topics, a complex structure, and extensive research. It is necessary to combine research contents from various disciplines in order to improve service usability, satisfaction, and efficiency, as well as to create common values for service providers and service recipients, in order to improve user experience and provide effective, feasible, efficient, and customer-expected services. The improved tourist destination bus query algorithm and the traditional algorithm are compared in Figure 6.

SOA is an ideal integration architecture. The rural tourism information service system is integrated based on

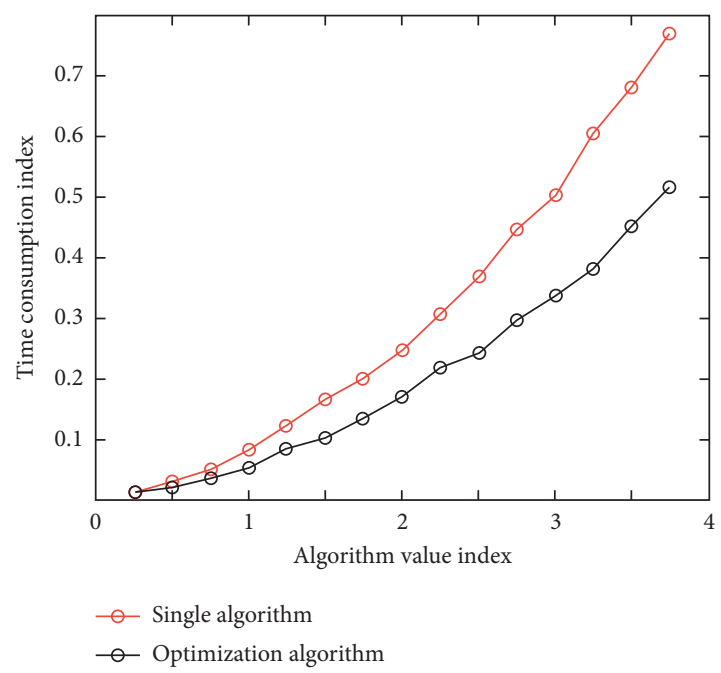

FIGURE 4: Comparison of algorithm's performance time.

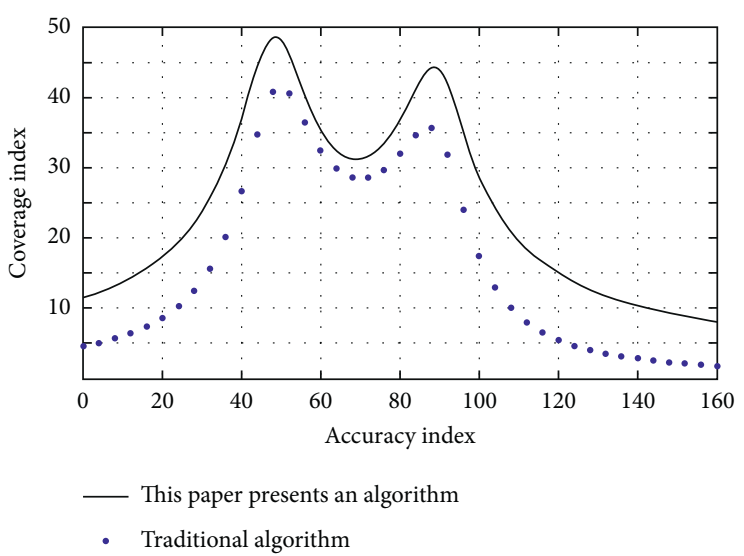

FIGURE 5: Comparison of accuracy and recall of the results obtained by two methods.

Web Services, which embodies the characteristics of loose coupling and independent protocol, can meet the needs of integration of various complex information systems, can support the dynamic business needs of packaging according to the needs, well solves the problems existing in the traditional system integration, and establishes a contact way of sharing integration for "information island." Study the classification performance of each scenic spot, and the result of single-level transfer learning model is shown in Figure 7.

To comply with the trend of personalized and personal tourism service industry, combine the benefits of the popularization and comprehensiveness of mobile Internet information, and build a network environment that provides customized experience and timely and effective access to information based on the essential characteristics and interests of users and other related factors, and propose a smart tourism experience evaluation system that can improve users' experiences. We compare the service system in this paper with the traditional service system in user satisfaction evaluation, and the comparison result is shown in Figure 8 . 


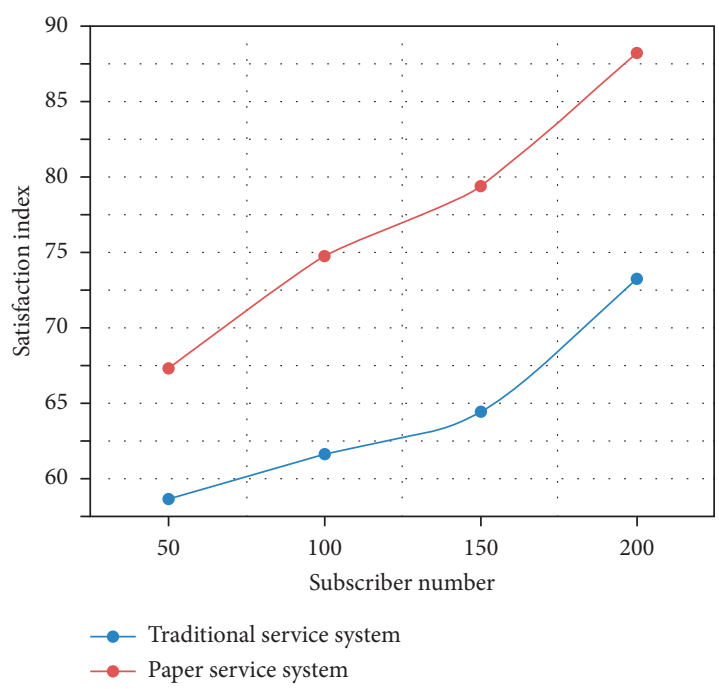

Figure 6: Comparison between the improved algorithm and the traditional algorithm.

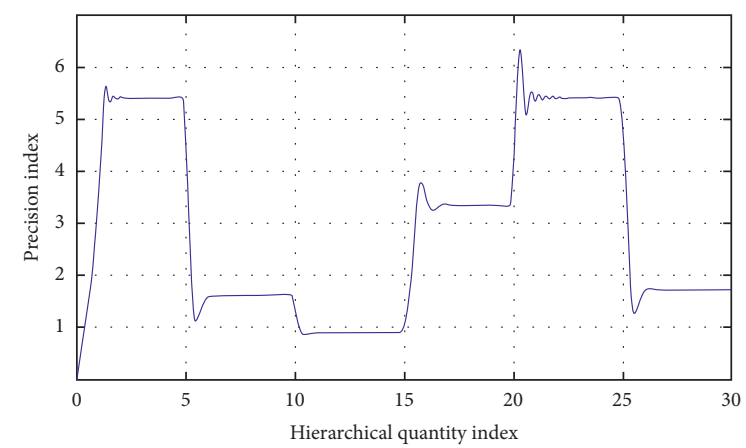

Figure 7: Data with different classification accuracy.

It is the basis of this data design process to accurately understand and analyze tourists' demand for bus travel information service in tourist destinations. The fundamental purpose of database design is to design an appropriate database according to tourists' needs and choose the appropriate database to ensure the platform can run at a high speed and stably. Through the analysis of tourists' demand and the research on the application background of the platform itself, we can see that this module needs the following data support: bus line information, bus station information, subway line information, subway station information, sign information near the station, and bus-to-subway information. Considering the saving of system resources at the same time, a cache information for storing the query results will be generated after the tourists finish the station query.

The overall architecture and technical architecture of the platform are designed by using SOA, and the working principle of the platform is expounded. According to the functional requirements of the platform, this paper analyzes the key implementation technologies of the platform's presentation layer, business logic layer, and data persistence layer. And debugging the system, the system has certain accuracy and practicability. Its main goal is to make tourists get high-quality services in the information service system

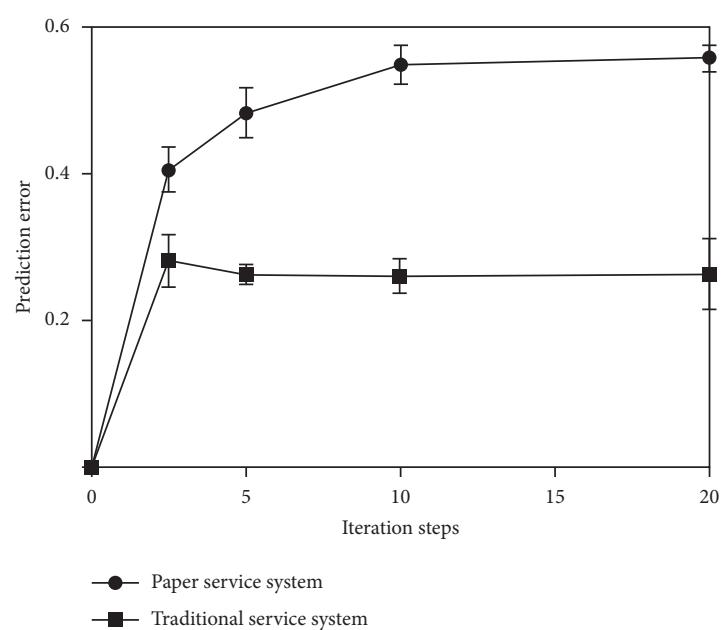

FIGURE 8: Comparison of user satisfaction evaluation.

and realize the convenience, personalization, and intelligence of tourism. At present, the tourism platform only provides simple information release and finished tourism routes, and there is an information island among service, management, marketing, and experience, which enhances the user experience.

\section{Conclusions}

Strengthening the informatization construction of rural tourism is the driving force for rural tourism to move forward and the guarantee for the sustainable development of rural tourism in the current situation of rapid development and allaround promotion of informatization. To ensure the sustainable development of rural tourism informatization, it is also necessary to strengthen the promotion of rural tourism informatization, expand network coverage, and constantly explore and innovate, in addition to expanding the informatization mode of rural tourism, strengthening the construction of websites, capital investment, publicity and education, personnel training, policies and regulations, and so on. Based on the tourism consumption model, according to tourists' user experience model and rural quality evaluation index system, in order to realize the personalized, intelligent, and convenient experience enjoyment of tourists in rural tourism, through user investigation and analysis of tourists' consumption behavior and psychology, based on the tourism consumption model and rural quality evaluation index system. The Web Service integration technology is primarily used in the service layer to eliminate differences in programming languages, platforms, protocols, and data structures. Project development is carried out under the network information service system in accordance with user experience requirements. We should tap the cultural connotation of rural scenic spots and make full use of rural local resources to create a high-quality experience service environment for tourists throughout the process, taking into account user needs and functional requirements of tourism projects, as well as network information technology and local characteristics of rural traditions. 


\section{Data Availability}

The data used to support the findings of this study are included within the article.

\section{Conflicts of Interest}

The author declares no conflicts of interest regarding the publication of this paper.

\section{References}

[1] V. Subramaniyaswamy, G. Manogaran, R. Logesh et al., "An ontology-driven personalized food recommendation in IoTbased healthcare system," The Journal of Supercomputing, vol. 75, no. 6, pp. 3184-3216, 2019.

[2] C. Zhang, "Design and application of fog computing and Internet of Things service platform for smart city," Future Generation Computer Systems, vol. 112, pp. 630-640, 2020.

[3] J. Zhang, J. Lu, and J. Peng, "Research on the management and control system of enterprise information service operation and maintenance based on SOA," Information/Communication, vol. 4, no. 3, 2016.

[4] G. Jin and N. Shao, "Development cost of water company information system based on SOA," Technology and Enterprise, vol. 1, no. 2, 2016.

[5] Y. Zhang, "Research and implementation of port information platform based on SOA architecture," Modern Economic Information, vol. 23, no. 2, 2019.

[6] X. Wang and Y. Duan, "Research on the informatization construction plan of surveying and mapping geographic information standard based on SOA," Standardization of Surveying and Mapping, 2017.

[7] Y. Tian and D. Wang, "Path selection for upgrading and transformation of rural tourism destinations in shandong province in the post-epidemic era," Humanities World, vol. 7, no. 6, 2020.

[8] J. Zhang and Y. Xing, "Opportunities, challenges and action strategies faced by rural tourism under the new crown epidemic," Liaoning Agricultural Sciences, vol. 6, no. 3, 2020.

[9] M. Chen and J. Gong, "The impact of the epidemic on rural tourism in Beijing and suggestions," Agricultural Products Market, vol. 19, no. 2, 2020.

[10] P. Yang, S. Li, and F. Yuan, "The impact of the new crown pneumonia epidemic on Shandong's agriculture and countermeasures," Rural Economy and Technology, vol. 31, no. 7, pp. 224-226, 2020.

[11] W. Liang, P. Huang, and C. Zhang, "Management innovation of superimposed management committee functions of village committees in rural tourism destinations from the perspective of epidemic prevention," Modern Management, vol. 11, no. 9, p. 8, 2021.

[12] Z. Bo, Z. Zheng, Z. Ren, J. Ma, and W. Wang, "Energy-efficient software-defined data collection by participatory sensing," IEEE Sensors Journal, vol. 16, no. 20, pp. 7315-7324, 2016.

[13] L. Peng, "Rural tourism in Pingdingshan City under the background of rural revitalization strategy," Northern Horticulture, vol. 14, pp. 197-200, 2018.

[14] R. K. Chigangaidze, "Risk factors and effects of the morbus: COVID-19 through the biopsychosocial model and ecological systems approach to social work practice," Social Work in Public Health, vol. 36, no. 2, pp. 1-20, 2020.
[15] S. Jiang, "Design of workflow informatization model based on SOA technology architecture," Engineering Technology, vol. 7, p. 289, 2016.

[16] L. Wang, "Research on the integrated development path of rural tourism information construction and smart tourism management," Tourism Overview, vol. 321, no. 12, pp. 46-47, 2020.

[17] D. Liu, "Conception of the construction of the Yangtze River hydrological data sharing service platform based on SOA," Water Resources and Hydropower Engineering, vol. 40, no. 1, p. 4, 2019.

[18] X. Hu and X. Zheng, "The design of a three-proof emergency command system based on SOA architecture," Digital Technology and Application, vol. 5, no. 1, 2016.

[19] L. Zhang, "The impact of the new crown pneumonia epidemic on rural tourism poverty alleviation and its response," Journal of Donghua University, vol. 36, no. 4, p. 5, 2020.

[20] C. Yun, "Research on new ideas of rural tourism poverty alleviation under the background of epidemic situation," Journal of Hubei Open Vocational College, vol. 33, no. 12, p. 3, 2020.

[21] C. Sun, "New media communication strategies for rural tourism under the influence of the new crown pneumonia epidemic," Journal of Kunming University of Science and Technology: Social Science Edition, vol. 21, no. 1, p. 9, 2021.

[22] Y. Ye and Y. Zheng, "The new situation of the development of rural tourism informatization under the smart tourism environment: taking Fujian Province as an example," Hubei Agricultural Sciences, vol. 55, no. 9, p. 4, 2016.

[23] P. Christou, A. Farmaki, and G. Evangelou, "Nurturing nostalgia?: a response from rural tourism stakeholders," Tourism Management, vol. 69, no. 12, pp. 42-51, 2018.

[24] Y. Yang, Z. Ding, and J. Ge, "Coupling and coordination relationship between rural tourism informatization and regional tourism economy in Jiangsu Province," Economic Geography, vol. 11, no. 6, 2018.

[25] W. Gao, Q. Zhang, Z. Lu, D. Wu, and X. Du, “Modelling and application of fuzzy adaptive minimum spanning tree in tourism agglomeration area division," Knowledge-Based Systems, vol. 143, no. 3, pp. 317-326, 2018.

[26] M. C. Olaverri, "Intelligent vehicle technology R\&D group [ITS research lab]," IEEE Intelligent Transportation Systems Magazine, vol. 10, no. 3, pp. 200-203, 2018.

[27] E. Dimitrakis, K. Sgontzos, and Y. Tzitzikas, "A survey on question answering systems over linked data and documents," Journal of Intelligent Information Systems, vol. 55, no. 5, pp. 1-27, 2019. 